\title{
Interferon-gamma as adjunctive immunotherapy for invasive fungal infections: a case series
}

\author{
Corine E Delsing ${ }^{1 \dagger}$, Mark S Gresnigt ${ }^{1 \dagger}$, Jenneke Leentjens ${ }^{1,2 \dagger}$, Frank Preijers ${ }^{4}$, Florence Allantaz Frager ${ }^{5}$, \\ Matthijs Kox ${ }^{2,3}$, Guillaume Monneret ${ }^{5}$, Fabienne Venet ${ }^{5}$, Chantal P Bleeker-Rovers ${ }^{1}$, Frank L van de Veerdonk ${ }^{1}$, \\ Peter Pickkers ${ }^{2}$, Alexandre Pachot ${ }^{5}$, Bart Jan Kullberg ${ }^{1}$ and Mihai G Netea ${ }^{1,6^{*}}$
}

\begin{abstract}
Background: Invasive fungal infections are very severe infections associated with high mortality rates, despite the availability of new classes of antifungal agents. Based on pathophysiological mechanisms and limited pre-clinical and clinical data, adjunctive immune-stimulatory therapy with interferon-gamma (IFN- $\gamma$ ) may represent a promising candidate to improve outcome of invasive fungal infections by enhancing host defence mechanisms.

Methods: In this open-label, prospective case series, we describe eight patients with invasive Candida and/or Aspergillus infections who were treated with recombinant IFN- $\gamma$ (rIFN- $-100 \mu$ g s.c., thrice a week) for 2 weeks in addition to standard antifungal therapy.

Results: Recombinant IFN- $\gamma$ treatment in patients with invasive Candida and/or Aspergillus infections partially restored immune function, as characterized by an increased HLA-DR expression in those patients with a baseline expression below $50 \%$, and an enhanced capacity of leukocytes from treated patients to produce proinflammatory cytokines involved in antifungal defence.
\end{abstract}

Conclusions: The present study provides evidence that adjunctive immunotherapy with IFN- $\gamma$ can restore immune function in fungal sepsis patients, warranting future clinical studies to assess its potential clinical benefit.

Trial registration: ClinicalTrials.gov - NCT01270490

Keywords: Immunotherapy, Interferon-gamma, Candidemia, Aspergillosis

\section{Background}

The incidence of fungal infections is steadily increasing in the last years due to invasive medical diagnosis and immunosuppressive treatment modalities. Despite development of new classes of antifungal agents [1], the invasive fungal infections remain associated with unacceptable high mortality rates and represent a major cause of death worldwide [2-7]. The emergence of significant resistance to the currently available antifungal therapies emphasizes the need for novel approaches to treat invasive fungal infections $[8,9]$. Invasive fungal infection are most commonly observed in individuals with immune defects or a

\footnotetext{
* Correspondence: mihai.netea@radboudumc.nl

${ }^{\dagger}$ Equal contributors

'Department of Internal Medicine, Division of Experimental Internal Medicine, Radboud University Medical Center, Nijmegen, The Netherlands

${ }^{6}$ Department of Internal Medicine (463), Radboud University Nijmegen Medical Center, P.O. Box 9101, 6500 HB Nijmegen, The Netherlands

Full list of author information is available at the end of the article
}

compromised immune system, and the number of these patients is steadily increasing [10]. Therefore, adjunctive immunotherapy to improve host defence is an attractive strategy to improve the outcome of patients with disseminated fungal infections.

In the past decade, major progress in the understanding of anti-fungal host responses has enabled the development of a number of novel molecular and cell-based immunotherapeutic approaches for invasive fungal infections [11]. Although invasive candidiasis and aspergillosis are rather different in their pathogenesis, the major protective host response against both fungi is the effective induction of Th1 and IFN- $\gamma$ responses [12-16]. The Th1 cytokine response activates effector phagocytic cells that kill the fungus [17]. Interestingly, Th1 immunity against $A$. fumigatus was demonstrated to be crossprotective against C. albicans [18]. 
Interferon-gamma (IFN- $\gamma)$, the prototype Th1 cytokine, promotes Th1 differentiation and skews the immune response towards a protective Th1 phenotype [19]. As such, it has been implicated as a treatment option in (invasive) fungal infections [20,21]. Moreover, limited evidence suggests that recombinant IFN- $\gamma$ (rIFN- $\gamma$ ) has a beneficial effect on the outcome of fungal infections in patients with chronic granulomatous disease (CGD) [22], HIV [23-25], leukemia [26,27], and in patients receiving organ transplants [28]. However, it has not been investigated whether rIFN- $\gamma$ actually enhances the immune response in these patients to explain these beneficial clinical effects.

In this report we describe a series of patients with invasive Candida and/or Aspergillus infections in whom we investigated the effects of treatment with $\mathrm{rIFN}-\gamma$ on the host innate and adaptive immune responses.

\section{Methods}

\section{Patients and treatment}

To assess the feasibility and preliminary efficacy of IFN- $\gamma$ in combination with anidulafungin for the treatment of candidemia, a single-centre, prospective, randomized open-label pilot (Phase IIIb) study was conducted. This study was registered at ClinicalTrials.gov (NCT01270490) and approved by the local ethics committee of the Radboud University Medical Center. Due to slower than anticipated enrollment rates (from August 2010 until March 2013, only 12 patients could be screened, of which 6 were eligible and provided informed consent [Figure 1]), the study was terminated early. However, during this period, several other patients presented with invasive fungal infections which had an insufficient response to standard antifungal therapy. Although these patients did not meet the inclusion criteria (i.e. presenting with one or more positive cultures of blood or normally sterile tissue growing Candida spp.), they were deemed to benefit from adjunctive immunotherapy as "therapy of last resort" as decided by the attending physician. Within the parameters of standard clinical care these patients were treated according to the same protocol as the patients enrolled in the study, and were therefore included in the present case series. All patients with a history of documented epileptic seizures, pre-existent severe renal impairment (creatinine clearance $<30 / \mathrm{mL} / \mathrm{min}$ ) or severe liver failure (defined as a spontaneously increased prothrombin time) were excluded. After obtaining informed consent, eight patients (3 study patients, 5 last resort patients) were treated with rIFN- $\gamma$ (Immukine, Boehringer Ingelheim, $50 \mu \mathrm{g} / \mathrm{m}^{2}$ body surface, subcutaneously, three times a week) in addition to standard antifungal therapy as recommended by national and international treatment guidelines $[29,30]$. Three patients who were included in the Phase IIIb Candida pilot-study were assigned to the control group and did not receive rIFN- $\gamma$.

\section{Blood sampling}

Plasma, serum and whole blood specimens were collected at baseline (BL) and serially after the start of antifungal therapy (days 1, 2, 3, 7, 14 and 28). Blood cultures were performed as part of routine care.

\section{Leukocyte populations and surface HLA-DR expression}

Heparin anticoagulated blood was stored at $4{ }^{\circ} \mathrm{C}$ immediately after withdrawal and analyzed by flow cytometry. To determine the extent of immune suppression, HLA-DR expression was determined by calculating \% HLADR-positive cells and HLA-DR mean fluorescence intensity (MFI) within $\mathrm{CD}_{14}{ }^{+}$cells and various lymphocyte subsets within $\mathrm{CD} 45^{+}$leukocytes ${ }^{+}$(see Additional file 1 and Additional file 2: Figure S1 for details and a representative flow diagram). Lymphocyte subsets were defined as: T-cells $\left(\mathrm{CD} 45^{+} \mathrm{CD}^{+}\right)$, T-helper cells (Th, $\mathrm{CD} 45^{+} \mathrm{CD}^{+} \mathrm{CD}^{+}$), cytotoxic T-cells (Tc, $\mathrm{CD} 45^{+} \mathrm{CD} 3^{+}$ $\left.\mathrm{CD} 8^{+}\right)$, B-cells $\left(\mathrm{CD} 45^{+} \mathrm{CD} 19^{+}\right)$, and NK-cells $\left(\mathrm{CD} 45^{+}\right.$ $\mathrm{CD}^{-} \mathrm{CD} 56^{+}$). Subset counts were calculated by multiplying the percentage of gated cells by the total lymphocyte count. Patients with $<50 \%$ HLA-DR positive monocytes at baseline were considered to exhibit immune paralysis. This threshold of 50\% is well below the lower bound of the $99 \%$ confidence interval obtained in healthy volunteers in an earlier study of our group using the same methodology in the same laboratory [31]. Therefore mHLA-DR expression levels below 50\% are likely to represent immunoparalysis.

\section{Cytokine assays}

Venous blood was drawn into $10 \mathrm{~mL}$ EDTA tubes, after which peripheral blood mononuclear cells (PBMCs) were isolated as described previously [32]. In short, blood was diluted in phosphate buffered saline (PBS) (1:1) and fractions were separated by Ficoll (Ficoll-Paque Plus, GE healthcare, Zeist, The Netherlands) density gradient centrifugation. Cells were washed twice with PBS and resuspended in RPMI-1640+ (RPMI-1640 Dutch modification supplemented with $10 \mu \mathrm{g} / \mathrm{mL}$ gentamicin, $10 \mathrm{mM} \mathrm{L}$-glutamine, and $10 \mathrm{mM}$ pyruvate) (Gibco, Invitrogen, Breda, The Netherlands). The PBMCs were counted using a particle counter (Beckmann Coulter, Woerden, The Netherlands) and were plated in 96 well round-bottom plates (Corning, NY, USA) at a final concentration of $2,5 \times 10^{6} / \mathrm{mL}$, in a total volume of $200 \mu \mathrm{L}$. The PBMCs were stimulated for 24 hours, 48 hours, and 7 days with medium alone, or medium containing E. coli lipopolysaccharide (LPS; $10 \mathrm{ng} / \mathrm{mL}$ ), phytohaemaglutinnin (PHA; $10 \mu \mathrm{g} / \mathrm{ml}$ ), heat-inactivated Candida albicans blastoconidia $\left(1 \times 10^{6} / \mathrm{ml}\right)$ or heat-inactivated Candida albicans hyphae (derived from $1 \times 10^{6} / \mathrm{m}$ conidia). After stimulation, cell culture supernatant was collected and stored at $-20^{\circ} \mathrm{C}$. When all samples were collected, 


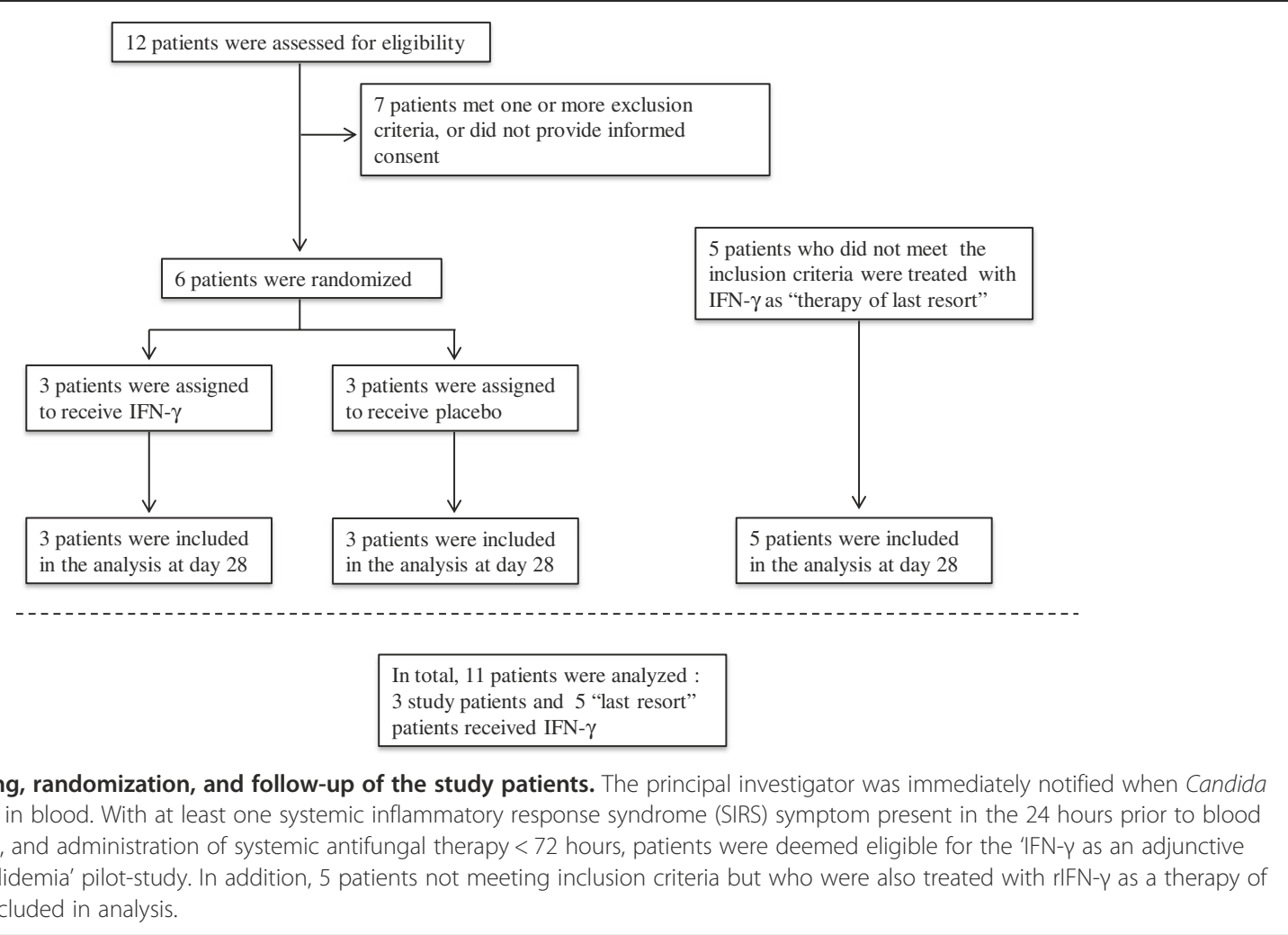

cytokines were measured using commercially available ELISAs (R\&D Systems, MN, USA and Sanquin, Amsterdam, The Netherlands) according to the protocols supplied by the manufacturer. Ex-vivo production of cytokines was assessed at timepoints at which their production has been shown to peak [33]. Monocyte derived cytokines such as Interleukin (IL)-1 $\beta$ and tumour necrosis factor (TNF) $\alpha$ were measured in culture supernatants of 24 hour cultures, IL-10 was measured in culture supernatants of 48 hour cultures. T-cell derived cytokines IL-17 and IL-22 were measured in culture supernatants of 7 day cultures.

\section{Statistical analysis}

In view of the small sample size, normality of distribution was not assumed. Comparisons of baseline with follow up time points were made using Wilcoxons signed rank test (within-group comparisons, 2 groups). A p-value of $<0.05$ was considered statistically significant. Data are expressed as means and standard error of the mean. Calculations and statistical analyses were performed using GraphPad Prism v 5.0 (GraphPad Software, San Diego, CA, USA).

\section{Results}

\section{Patient characteristics}

The patients treated with rIFN- $\gamma$ ( 5 men, 3 women) had a median age of 49.5 [IQR 28.5-68.8] years. The three female patients in the control group were 36, 51 and 73 years old. Clinical characteristics of the participants are listed in Table 1 . Of the 6 patients included in the pilot study, three patients had a positive blood culture for C. albicans, two patients for C. glabrata, and one patient for C. tropicalis. During randomization, the three patients with $C$. albicans cultures were assigned to rIFN- $\gamma$ treatment, whereas the two patients with $C$. glabrata and one with $C$. tropicalis cultures were assigned to the control group. However, no pathophysiological evidence currently exists to suggest that $\operatorname{rIFN}-\gamma$ therapy would have a different effect on the immune system in case of albicans vs. non-albicans Candida infections. Of the other 5 patients treated with rIFN- $\gamma$ as therapy of last resort, one patient had proven acute aspergillosis, one had probable acute aspergillosis, and one probable chronic aspergillosis according to the EORTC/MSG criteria [34]. One patient had a positive blood culture for C. tropicalis associated with osteomyelitis. This patient developed new suspected lesions on positron emission tomography-computed tomography (PET-CT) while receiving antifungal treatment. In another patient, CTscan revealed progression of suspected hepatic Candida lesions during antifungal treatment. All patients included suffered some degree of immunosuppression: the 6 patients with positive blood cultures for Candida spp. had impaired physical barriers due to the presence of 
Table 1 Summary of clinical characteristics of all patients with invasive fungal infections

\begin{tabular}{|c|c|c|c|c|c|c|c|c|c|c|}
\hline \multicolumn{11}{|c|}{ IFN- $\gamma$ treated patients } \\
\hline Age & Pathogens & $n=$ & Site of nfection & $n=$ & Underlying illness & $n=$ & Antifungal therapy & $\mathrm{n}=$ & Outcome & $n=$ \\
\hline $49.6 \pm S D 19.8$ & C. albicans & 3 & Candidaemia & 2 & Stem cell transplantation for AML & 1 & Anidulafungin & 1 & $\begin{array}{l}\text { Cured without further infectious } \\
\text { complications }\end{array}$ & 2 \\
\hline BMI & Candida spp & 2 & $\begin{array}{l}\text { Candidaemia } \\
\text { Endocarditis }\end{array}$ & 1 & $\begin{array}{l}\text { Sarcoidosis treated with prednisone } \\
\text { and azathioprin }\end{array}$ & 1 & Fluconazole & 1 & $\begin{array}{l}\text { Lost to follow up after discharge } \\
\text { from hospital }\end{array}$ & 1 \\
\hline $22.9 \pm S D 6.9$ & A. fumigatus & 2 & $\begin{array}{l}\text { Pulmonary } \\
\text { aspergilllosis }\end{array}$ & 3 & $\begin{array}{l}\text { First remission induction chemotherapy } \\
\text { for AML }\end{array}$ & 1 & L-AMB + Voriconazole & 1 & Slight reduction hepatic lesions & 1 \\
\hline Gender & C. tropicalis & 1 & Osteomeyelitis & 1 & $\begin{array}{l}\text { ICD, Streptococcus sanguis endocarditis, } \\
\text { aorta valve replacement with bioprosthesis }\end{array}$ & 1 & $\begin{array}{l}\text { Voriconazole + } \\
\text { Anidulafungin }\end{array}$ & 1 & $\begin{array}{l}\text { Cured but complicated with } \\
\text { mycotic cerebral aneurysms }\end{array}$ & 1 \\
\hline F: 5 M: 3 & $\begin{array}{l}\text { A. fumigatust } \\
\text { M. genavese }\end{array}$ & 1 & Hepatic abcess & 1 & $\begin{array}{l}\text { persistent pulmonary cavity after radiotherapy } \\
\text { for a T1NOMO lungcarcinoma }\end{array}$ & 1 & $\begin{array}{l}\text { Itraconasole, L-AMB, } \\
\quad \text { Voriconazole }\end{array}$ & 1 & $\begin{array}{l}\text { Cured from candidemia episode, } \\
4 \text { months later unrelated bacterial } \\
\text { sepsis episode }\end{array}$ & 1 \\
\hline & & & & & $\begin{array}{c}\text { Total parenteral nutrition via Hickmann catheter } \\
\text { because of slow transit bowel, intestinal pseudo } \\
\text { obstruction, or gastroparesis }\end{array}$ & 3 & $\begin{array}{l}\text { Anidulafungin and step } \\
\text { down to fluconazol }\end{array}$ & 3 & $\begin{array}{l}\text { Died due to infectious complications } \\
71 \text { or } 15 \text { days after initiation of } \\
\text { IFN- } y \text { therapy }\end{array}$ & 2 \\
\hline \multicolumn{11}{|c|}{$\begin{array}{ll}\text { Placebo treated patients } \\
\end{array}$} \\
\hline $53.0 \pm$ SD19.1 & C. glabrata & 2 & Candidaemia & 3 & $\begin{array}{l}\text { Total parenteral nutrition via Hickmann } \\
\text { catheter because of slow transit bowel }\end{array}$ & 1 & Anidulafungin & 2 & $\begin{array}{l}\text { Cured without further infectious } \\
\text { complications }\end{array}$ & 3 \\
\hline BMI & C. tropicalis & 1 & & & HIV with porth-a-cath for venous access & 1 & $\begin{array}{l}\text { Anidulafungin }+ \\
\text { amphotericin B }\end{array}$ & 1 & & \\
\hline $18.5 \pm \mathrm{SD} 4.0$ & & & & & $\begin{array}{l}\text { construction of ileal conduit urinary } \\
\text { diversion (Bricker deviation) because of } \\
\text { pT4N2M1 bladder cancer. }\end{array}$ & 1 & & & & \\
\hline \multicolumn{11}{|l|}{ Gender } \\
\hline F: 3 M: 0 & & & & & & & & & & \\
\hline
\end{tabular}


indwelling venous catheters (for the need of recurrent blood sampling or total parental nutrition), or an implantable cardioverter-defribillator (ICD) lead. The patient with progression of suspected hepatic Candida lesions on the CT-scan, and one patient with proven acute aspergillosis (diagnosed by isolation of $A$. fumigatus from lung tissue biopsies) were immunocompromised because of (therapy for) acute myeloid leukaemia. Another patient with acute invasive aspergillosis (A. fumigatus isolated from BAL fluid) received immunosuppressive therapy (prednisone and azathioprine) for sarcoidosis and suffered from a coinfection with Mycobacterium genavense localized in the bone marrow. A third patient developed chronic pulmonary aspergillosis (diagnosed by consistent $\mathrm{CT}$ abnormalities with cavitation and culture of $A$. fumigatus from BAL fluid) after radiotherapy for lung carcinoma. Only two patients, both with acute aspergillosis, were admitted to the Intensive Care Unit to receive organ supportive therapy (mechanical ventilation and hemodynamic support).

\section{Clinical outcome}

The three patients in the control group and five out of eight patients treated with rIFN- $\gamma$ recovered uneventfully from the fungal infection (Table 1). Two patients with invasive aspergillosis that were already admitted to the ICU at the time of treatment died due to infectious complications of severe pulmonary aspergillosis, despite rIFN- $\gamma$ treatment. The patient with a Candida endocarditis, who despite rIFN- $\gamma$ treatment developed intracerebral mycotic aneurysm, could be discharged from the hospital 93 days after onset of invasive candidiasis.

In all patients treated, rIFN- $\gamma$ was well tolerated. Five patients reported moderate fever upon administration of rIFN- $\gamma$, which responded well to acetaminophen. Two patients developed liver enzyme abnormalities for which tuberculostatic antibiotics and voriconazole were temporarily discontinued, resulting in recovery of the liver enzyme abnormalities while $\mathrm{rIFN}-\gamma$ treatment was continued. No other significant adverse events were observed.

\section{Effect of rIFN- $\gamma$ on ex-vivo IL-1 $\beta$ and TNFa production}

To assess the effect of rIFN- $\gamma$ on the capacity of PBMCs to produce pro-inflammatory cytokines, cells were isolated and stimulated before, during, and after treatment. We monitored the fold change in cytokine production compared with baseline (before start of treatment). IL- $1 \beta$ and TNF $\alpha$ are pro-inflammatory cytokines of the innate immune system crucial in the induction and maintenance of the anti-fungal immune response [35-40]. Before IFN- $\gamma$ treatment, inter-patient variability in cytokine production was high (e.g. TNF $\alpha$ median [IQR] concentration after stimulation with LPS was $792 \mathrm{pg} / \mathrm{mL}$ [314-2005]). Nevertheless, in all patients an increase in the capacity to induce different cytokines was observed in the first two days after initiation of IFN- $\gamma$ treatment, independent of their baseline values (group data shown in Figure 2), at subsequent time points only a trend towards increased could be observed. In contrast, the placebo-treated patients IL- $1 \beta$ and TNF $\alpha$ responses over time remained similar to baseline. The response against hyphae of $C$. albicans was highly variable between patients. Some rIFN- $\gamma$-treated patients demonstrated a profound increase of TNF $\alpha$ production after treatment (up to 70 fold), whereas other patients showed no relevant change in TNF $\alpha$ production. Cytokine production remained similar in patients in the control group.

\section{Effect of rIFN- $\gamma$ on ex-vivo IL-17 and IL-22 production}

Both IL-17 and IL-22 are cytokines that are thought to be protective in the host defence against invasive fungal infections [35,41-45]. PHA-induced IL-17 and IL-22 production was increased 1 day after initiation of rIFN- $\gamma$ treatment (Figure 3). However, at subsequent time points a trend towards increased IL-17 and IL-22 production was observed, which reverted to baseline levels at day 28. Production of IL-17 and IL-22 upon stimulation with Candida blastoconidia was elevated after rIFN- $\gamma$ treatment in 6 of 8 patients. Hyphae induced IL-17 and IL-22 production was increased in 4 of 8 and 5 of 8 patients respectively. Patients who received placebo therapy did not display a trend towards increased IL-17 or IL-22 production during the course of treatment (group data shown in Figure 3).

\section{Effect of rIFN- $\gamma$ on ex-vivo IL-10 production}

In addition to pro-inflammatory cytokines, the capacity to produce anti-inflammatory cytokines can also influence disease outcome. In particular the anti-inflammatory cytokine IL-10 has been associated with protection against immunopathology during severe infections. IL-10 production in response to stimulation with LPS, PHA and Candida was highly variable between patients and did not show a distinct pattern following rIFN- $\gamma$ treatment (Figure 4). No relevant differences compared to the placebo-treated patients were observed.

\section{HLA-DR expression}

The numbers of HLA-DR-positive monocytes, a marker of immunosuppression, varied substantially between patients at baseline (39.05\% [27.5-61.6] vs. 90.6 [88.7-92.5] in IFN- $\gamma$-treated patients and placebo-treated patients, respectively). Five out of eight IFN- $\gamma$ treated patients exhibited HLA-DR positive monocyte levels below the "immunoparalysis threshold" of $50 \%$ and in these patients, an increase of HLA-DR-positive monocytes after IFN- $\gamma$ treatment between $10 \%$ and $44 \%$ was observed which persisted throughout the study period (Figure 5). 


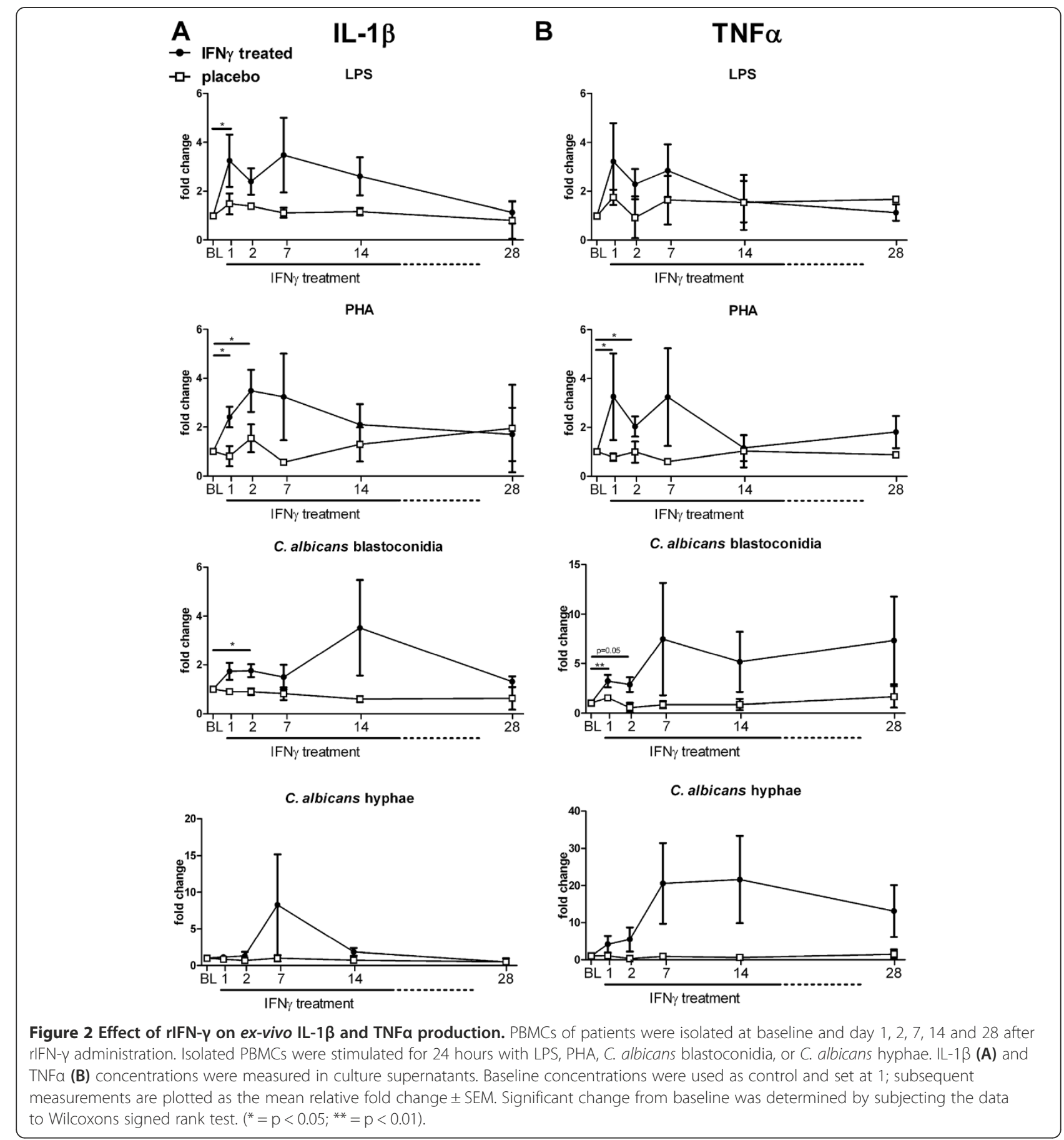

Patients with a baseline HLA-DR expression higher than $50 \%$ did not show a change in expression. The patient with a HLA-DR-expression $<50 \%$ who did not show increased levels of HLA-DR positive monocyte numbers at any time point, was one of the two patients who died due to infectious complications. No correlation was found between the level of mHLA-DR expression and TNF $\alpha$ production of LPS-stimulated PBMCs. An inverse correlation of baseline mHLA-DR levels with severity of underlying illness and tissue involvement was found (with higher mHLA-DR levels in patients with only impaired physical barriers, e.g. due to indwelling catheters, compared to patients with impaired immune responses, e.g. due to chemotherapy, immune suppressive agents, bone marrow disease; data not shown because this compromises patients anonymity). 

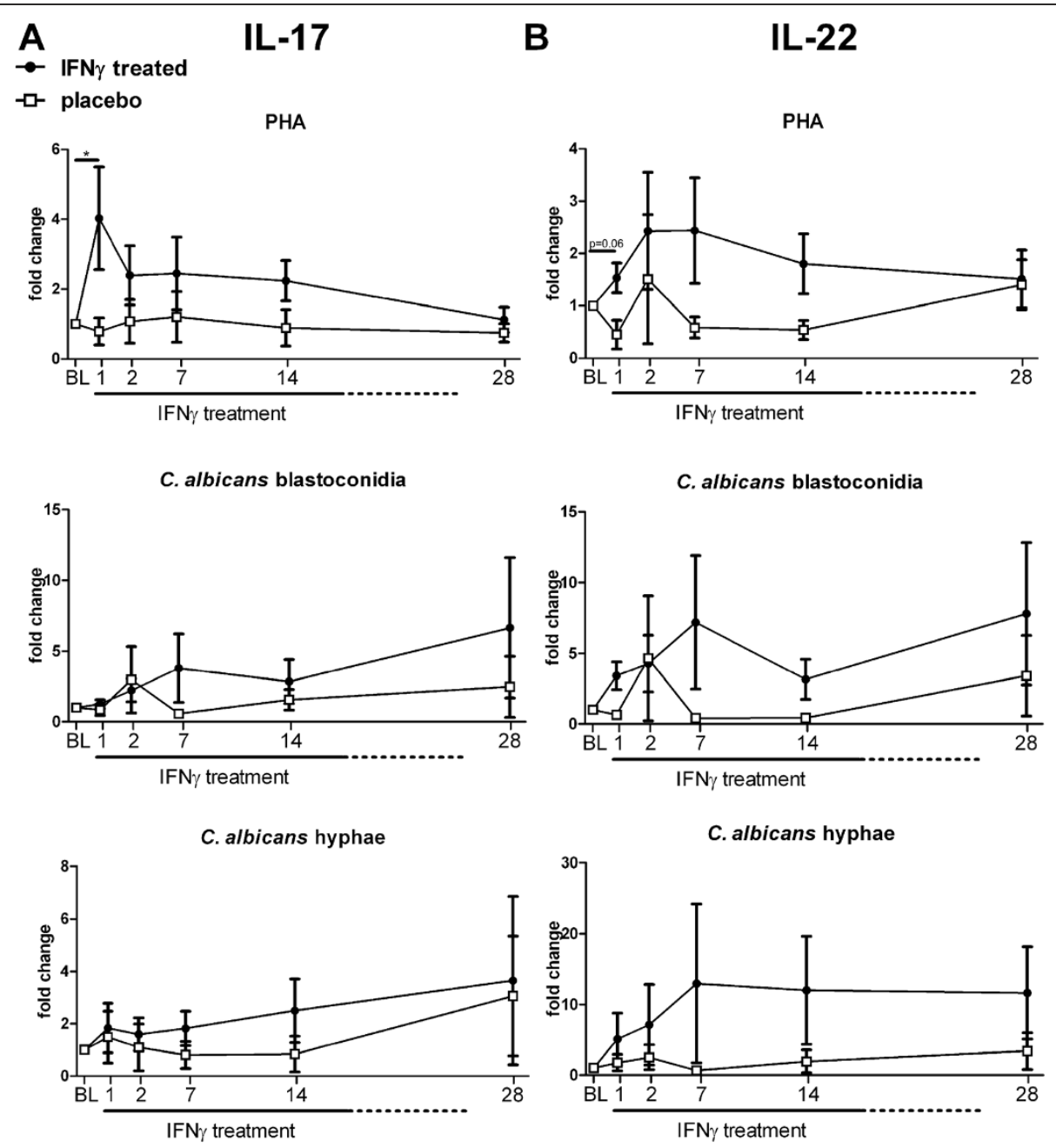

Figure 3 Effect of rIFN- $\gamma$ on ex-vivo IL-17 and IL-22 production. PBMCs of patients were isolated at baseline and day 1, 2, 7, 14 and 28 after rIFN- $\gamma$ administration. Isolated PBMCs were stimulated for 7 days with PHA, C. albicans blastoconidia, or C. albicans hyphae. IL-17 (A) and IL-22 (B) concentrations were measured in culture supernatants. Baseline concentrations were used as control and set at 1; subsequent measurements are plotted as the mean relative fold change \pm SEM. Significant change from baseline was determined by subjecting the data to Wilcoxons signed rank test. $\left(^{*}=p<0.05\right)$.

\section{Cell populations}

There were no significant changes in the total leukocyte and granulocyte numbers in rIFN- $\gamma$-treated patients (Additional file 3: Figure S2A). Monocyte counts significantly increased one week after initiation of rIFN- $\gamma$ therapy (Additional file 3: Figure S2C) and lymphocyte numbers significantly increased at 2 and 7 days after initiation of rIFN- $\gamma$ therapy (Additional file 3: Figure S2D), which could be attributed to slight changes in CD4 lymphocytes (Additional file 3: Figure S2E), B-lymphocyte (Additional file 3: Figure S2F) and NK-cell numbers (Additional file 3: Figure S2G) and a significant increase of CD8 lymphocytes (Additional file 3: Figure S2H). No clear changes in leukocyte (subset) counts were observed in placebo-treated patients.

\section{Discussion and conclusions}

While several small clinical trials illustrated the beneficial clinical effects of adjuvant treatment with IFN-y, the proposed immunostimulating effect of IFN- $\gamma$ as the mechanism of action has not been investigated. In this case series we demonstrate for the first time that adjunctive immunotherapy with $\mathrm{rIFN}-\gamma$ improves the leukocyte immune responses in patients with severe invasive fungal infections. This was primarily reflected by increased ex-vivo pro-inflammatory cytokine responses of the innate immune system such as IL- $1 \beta$ or TNF $\alpha$, as well as an increased production of the T-cell cytokines IL-17 and IL-22, which are known to play an important role in the anti-fungal host defence [35,41-45], and by an increase in HLA-DR expression in mHLA-DR 


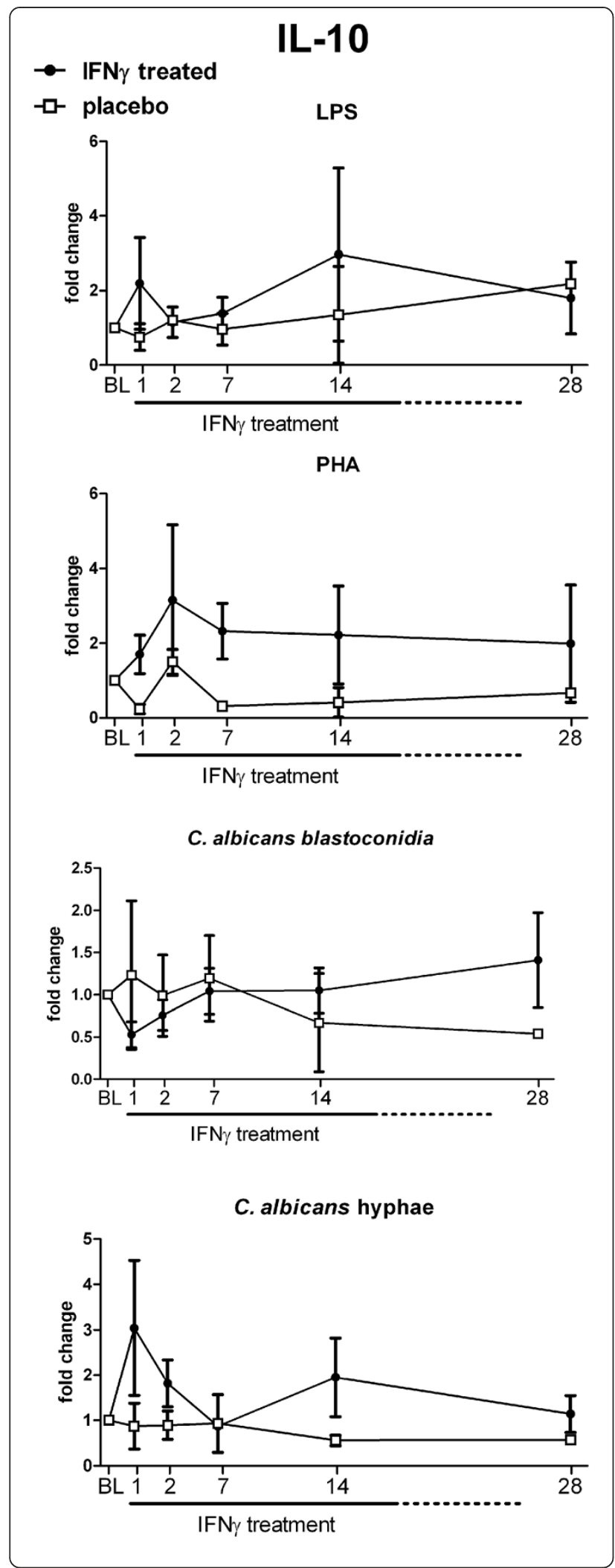

Figure 4 Effect of rIFN- $\gamma$ on ex-vivo IL-10 production. PBMCs of patients were isolated at baseline and day 1, 2, 7, 14 and 28 after rIFN- $\gamma$ administration. Isolated PBMCs were stimulated for 48 hours with LPS, PHA, C. albicans blastoconidia, or C. albicans hyphae. IL-10 concentrations were measured in culture supernatants. Baseline concentrations were used as control and set at 1; subsequent measurements are plotted as the mean relative fold change \pm SEM.

expression in those patients with a low cellular expression as a measure of their immune suppression.

In addition to enhanced ex-vivo responses, subtle changes in the leukocyte differentiation were observed following IFN- $\gamma$ treatment. Although there were no significant differences in total leukocyte numbers after treatment with rIFN- $\gamma$, shifts in leukocyte subpopulations such as increased monocyte and lymphocyte counts were apparent. While lymphocyte numbers increased after rIFN- $\gamma$ therapy, it could not directly be attributed to a specific subset as all of them showed increased values. The most significant increase was that of $\mathrm{CD} 8$ cells one week after initiation of rIFN- $\gamma$ therapy. Monocytes and lymphocytes are known to be crucial cells in the host defence against fungal infections. However, the increase of monocytes and lymphocytes during $\operatorname{rIFN}-\gamma$ therapy was accompanied by slightly decreased circulating granulocyte numbers. It is not known whether this reduction is due to activation and migration into the infected tissue, or whether a true decrease in granulocyte generation was induced by the treatment. Although the decrease in granulocyte numbers was slight, the fact that granulocytes, and especially neutrophils, are crucial in the antifungal host defence warrant careful monitoring of granulocyte numbers during IFN- $\gamma$ treatment.

Several clinical studies and case reports have previously demonstrated beneficial effects of rIFN- $\gamma$ in combination with antifungal therapy on outcome of fungal infections (for example in patients with CGD $(n=130)$ [22,46,47], HIV $(n=173)$ [23-25], leukaemia $(n=5)$ $[26,27]$, and transplant patients $(n=7)$ [28], in a patient with $S$. aureus liver abscess and invasive $C$. albicans infection [48], in a patient with intracerebral aspergillosis [49], in two patients with progressive chronic pulmonary aspergillosis [50], and in two patients with idiopathic CD4 lymphopenia and cryptococcal meningitis [51]). However, in contrast to our study, ex-vivo immune responses in these patients were not investigated. Due to the limited number of patients and the very heterogeneous population, we could not assess clinical endpoints, although a mean mortality of $25 \%$ in the IFN- $\gamma$ treated patients lies below the mean $40 \%$ estimated in patients with invasive fungal infections $[10,52]$.

To the best of our knowledge, we are the first to describe mHLA-DR expression, a widely used marker of 


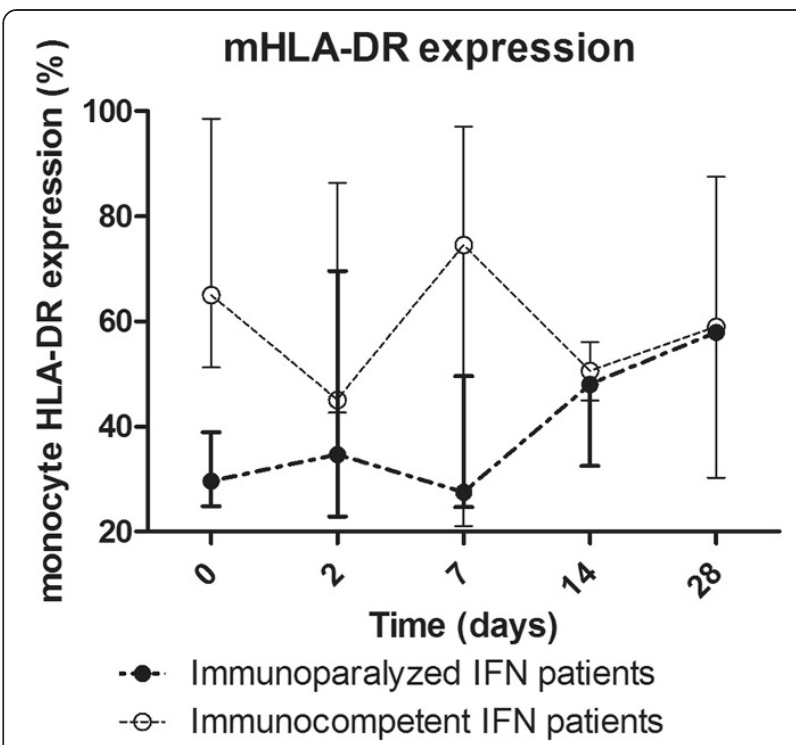

Figure 5 mHLA-DR expression in rIFN- $\gamma$ treated patients, divided into immunoparalyzed patients with baseline HLA-DR expression below $50 \%$ (solid dots), and without HLA-DR defined immunoparalysis (open dots). Data are expressed as median [IQR].

immunosuppression in (bacterial) sepsis patients [53], in patients with invasive fungal infections. In all IFN- $\gamma$ treated patients who showed baseline mHLA-DR levels below the immunoparalysis threshold of $50 \%$ and survived, IFN- $\gamma$ mediated upregulation of mHLA-DR expression was observed. In agreement with the data presented in this case series, $\operatorname{rIFN}-\gamma$ has been shown to significantly increase numbers of HLA-DR-positive monocytes both in a human preclinical bacterial sepsis model and in septic patients [31,54]. Reduced production of TNF $\alpha$ by leukocytes ex-vivo stimulated with LPS has also been shown to be marker of immunoparalysis in sepsis patients. In contrast to our study, mHLA-DR expression and ex-vivo TNF $\alpha$ production were found to be highly correlated in bacterial sepsis patients $[54,55]$. A possible explanation for this discrepancy is that, in contrast with the emerging consensus that immunoparalysis renders patients more vulnerable to opportunistic infections in general [53], different defects in immune defences may be responsible for enhanced susceptibility towards different pathogens.

Based on the apparent inverse correlation of baseline mHLA-DR levels with severity of underlying illness and tissue involvement, mHLA-DR levels seem to reflect disease severity and general immune status, and not specific immune defects per se. Hence, patients with invasive fungal infections and associated impaired antifungal immune responses will probably benefit more from immunostimulatory treatment compared to patients with only impaired physical barriers, e.g. due to indwelling catheters and apparent intact anti-fungal immune responses. Biomarkers reflecting the capacity of specific anti-fungal immune defences are required to identify patients who suffer from invasive fungal infections due to impaired cell-mediated immunity. It is important to identify such patients and attempt a tailored immunotherapeutic approach guided by the actual level and type of immunoparalysis of that specific patient. A blood based assay has been described that demonstrates a failure to induce IFN- $\gamma$ expression in renal transplant patients and differences in IL-10 and TNF $\alpha$ expression [56], which could be promising biomarkers to identify patients who could benefit from adjunctive immunotherapy.

The intracellular mechanism(s) through which the beneficial effects of IFN- $\gamma$ are mediated remain to be elucidated. Recently it was proposed that IFN- $\gamma$ exerts its effects at the transcription level [57], while others have demonstrated that IFN- $\gamma$ reverses tolerance-associated epigenetic modifications [58]. Another possible mechanism involved in the IFN- $\gamma$ mediated reversal of immunoparalysis is the downregulation of negative TLR regulators such as IRAK-M, a protein that negatively regulates LPSinduced inflammatory responses and contributes to the development of immunoparalysis [59].

Administration of rIFN- $\gamma$ was tolerated well. Several patients developed a mild fever upon administration, which responded well to acetaminophen treatment. No other side effects were observed. The most important limitation of the present study is the limited number of patients studied. Because the control group consisted of only three patients, no statistical analysis between the treatment and control groups could be performed. However, despite the small sample size, the increase in HLADR expression in patients with mHLA-DR expression levels below $50 \%$ and the increased ex-vivo response of several cytokines that are crucial in antifungal host defence is a promising observation that underlines the potential of immunotherapy. The slow enrolment of patients presenting with candidemia was the main factor contributing to the decision to terminate the phase IIIb Candida pilot-study early. With a reported incidence of 2.5-11 per 100,000 persons in Europe [60], and based on previous epidemiological data in our hospital this low enrollment was not expected at the time of the initiation of the study. The much lower incidence of candidemia in the last two years in our hospital is most likely due to a new antibiotic stewardship introduced recently in our hospital, which has reduced the incidence of opportunistic infections. The cut-off value of mHLA-DR expression levels of $50 \%$ to distinguish between immunoparalyzed and immunocompetent patients is another limitation of this study, as this is an arbitrary value chosen. We chose this value because it is well below the 99\% CI of mHLADR values in healthy volunteers [31]. Therefore, patients with mHLA-DR below $50 \%$ do have an impaired antigen 
presenting capacity of their monocytes which we show to be enhanced by IFN- $\gamma$ therapy. Whether this cut-off value truly represents immunoparalysis, reflected by enhanced susceptibility to secondary infections or reduced capacity to clear opportunistic infections, remains to be investigated. Furthermore, the use of a standardized analysis technique to quantify mHLA-DR, such as the Quantibrite method, is preferable, because it facilitates an objective comparison of mHLA-DR expression levels between studies and aids in the definitive establishment of a cut-off value to identify immunoparalyzed patients. Larger studies are required to confirm the data obtained here. To do so, multicentre studies should be facilitated in order to fully explore the potential of IFN- $\gamma$ immunotherapy.

Our data indicate that adjunctive immunotherapy with rIFN- $\gamma$ in patients with invasive fungal infections partially restores cell-mediated immunity. This suggests that IFN- $\gamma$ treatment enhances anti-fungal immunity and larger studies are warranted to validate the findings reported here and to assess the impact of IFN $-\gamma$ treatment on clinical outcome. Biomarkers of impaired anti-fungal immunity should be further investigated in order to identify patients who will benefit most from immunostimulatory therapy.

\section{Additional files}

Additional file 1: Online Supplement Flow cytometric analysis of mHLA-DR expression and lymphocyte subset counts.

Additional file 2: Figure S1. Representative flow diagram of monocyte HLA-DR measurements. Heparin blood was first analysed on forward- and side scatter to exclude cell debris and erythrocytes (A). Subsequently, $\mathrm{CD} 45^{+}$cells were selected (B) and within the $\mathrm{CD} 45^{+}$fraction was gated for $\mathrm{CD} 14^{+}$cells (C). The $\mathrm{CD} 45^{+} \mathrm{CD} 14^{+}$cells (D) were analysed for the percentage of HLA-DR positivity (E).

Additional file 3: Figure S2. Changes in immune cell populations. Total leukocyte numbers (A) and numbers of granulocytes (B), monocytes (C) and lymphocytes (D) measured in peripheral blood. Numbers of CD4 lymphocytes (E), B-lymphocytes (F), CD8 lymphocytes (G) and NK cells (H) within the lymphocyte population were quantified using flowcytytometry

\section{Competing interests}

None of the authors have any competing interests regarding this study. The support for the immunological assessments in this study was provided by an unrestricted grant from BioMérieux.

\section{Authors' contributions}

$C D, P P, A P, B J K$ and $M N$ conceived and designed the study. CD, JL and CBR screened and included patients. MG and FF carried out in-vitro experiments. $C D, M G, J L, F V$ and $M K$ analysed the data. MG, JL, FP. MK, PP, GM, AP and $M N$ participated in the data interpretation. $C D, M G$, $J$ wrote the manuscript draft. $M G, J L, M K, P P, B J K$ and $M N$ contributed to writing the final manuscript. All authors read and approved the final manuscript.

\section{Acknowledgements}

This research was performed within the framework of CTMM, the Center for Translational Molecular Medicine (www.ctmm.nl), project MARS (grant 04I-201). M.G.N. was supported by a Vici grant of the Netherlands Organization for Scientific Research and an ERC Consolidator grant of the European Research Council (nr. 310372). F.vd.V. was supported by a Veni grant of the Netherlands Organization for Scientific Research.

\section{Author details}

'Department of Internal Medicine, Division of Experimental Internal Medicine, Radboud University Medical Center, Nijmegen, The Netherlands.

${ }^{2}$ Department of Intensive Care Medicine, Radboud University Medical Center, Nijmegen, The Netherlands. ${ }^{3}$ Department of Anaesthesiology, Radboud University Medical Center, Nijmegen, The Netherlands. ${ }^{4}$ Department of Laboratory Medicine, Laboratory of Hematology, Radboud University Medical Center, Nijmegen, The Netherlands. ${ }^{5}$ Joint Unit « Sepsis » Hospices Civils de Lyon-bioMérieux, Hôpital Edouard Herriot, Lyon, France. ${ }^{6}$ Department of Internal Medicine (463), Radboud University Nijmegen Medical Center, P.O. Box 9101, 6500 HB Nijmegen, The Netherlands.

Received: 21 October 2013 Accepted: 14 March 2014

Published: 26 March 2014

\section{References}

1. Segal BH, Steinbach WJ: Combination antifungals: an update. Expert Rev Anti Infect Ther 2007, 5(5):883-892.

2. Wisplinghoff $H$, Bischoff T, Tallent SM, Seifert $H$, Wenzel RP, Edmond MB: Nosocomial bloodstream infections in US hospitals: analysis of 24,179 cases from a prospective nationwide surveillance study. Clin Infect Dis 2004, 39(3):309-317.

3. Gudlaugsson O, Gillespie S, Lee K, Vande Berg J, Hu J, Messer S, Herwaldt L, Pfaller M, Diekema D: Attributable mortality of nosocomial candidemia, revisited. Clin Infect Dis 2003, 37(9):1172-1177.

4. Horn DL, Neofytos D, Anaissie EJ, Fishman JA, Steinbach WJ, Olyaei AJ, Marr KA, Pfaller MA, Chang CH, Webster KM: Epidemiology and outcomes of candidemia in 2019 patients: data from the prospective antifungal therapy alliance registry. Clin Infect Dis 2009, 48(12):1695-1703.

5. Zaoutis TE, Argon J, Chu J, Berlin JA, Walsh TJ, Feudtner C: The epidemiology and attributable outcomes of candidemia in adults and children hospitalized in the United States: a propensity analysis. Clin Infect Dis 2005, 41(9):1232-1239.

6. Warnock DW: Trends in the epidemiology of invasive fungal infections. Nippon Ishinkin Gakkai Zasshi 2007, 48(1):1-12.

7. Brown GD, Denning DW, Gow NA, Levitz SM, Netea MG, White TC: Hidden killers: human fungal infections. Sci Trans/ Med 2012, 4(165):165rv113.

8. Rodloff C, Koch D, Schaumann R: Epidemiology and antifungal resistance in invasive candidiasis. Eur J Med Res 2011, 16(4):187-195.

9. Howard SJ, Cerar D, Anderson MJ, Albarrag A, Fisher MC, Pasqualotto AC, Laverdiere M, Arendrup MC, Perlin DS, Denning DW: Frequency and evolution of Azole resistance in Aspergillus fumigatus associated with treatment failure. Emerg Infect Dis 2009, 15(7):1068-1076.

10. Kriengkauykiat J, Ito Jl, Dadwal SS: Epidemiology and treatment approaches in management of invasive fungal infections. Clin Epidemiol 2011, 3:175-191.

11. Romani L: Immunity to fungal infections. Nat Rev 2011, 11(4):275-288.

12. Cenci E, Mencacci A, Del Sero G, Bistoni F, Romani L: Induction of protective Th1 responses to Candida albicans by antifungal therapy alone or in combination with an interleukin-4 antagonist. J Infect Dis 1997, 176(1):217-226.

13. Netea MG, Vonk AG, van den Hoven M, Verschueren I, Joosten LA, van Krieken JH, van den Berg WB, Van der Meer JW, Kullberg BJ: Differential role of IL-18 and IL-12 in the host defense against disseminated Candida albicans infection. Eur I Immunol 2003, 33(12):3409-3417.

14. Chai LY, van de Veerdonk F, Marijnissen RJ, Cheng SC, Khoo AL, Hectors M, Lagrou K, Vonk AG, Maertens J, Joosten LA, Kullberg BJ, Netea MG: Anti-Aspergillus human host defence relies on type $1 \mathrm{~T}$ helper (Th1), rather than type $17 \mathrm{~T}$ helper (Th17), cellular immunity. Immunology 2010, 130(1):46-54.

15. Cenci E, Mencacci A, Del Sero G, Bacci A, Montagnoli C, d'Ostiani CF, Mosci P, Bachmann M, Bistoni F, Kopf M, Romani L: Interleukin-4 causes susceptibility to invasive pulmonary aspergillosis through suppression of protective type I responses. J Infect Dis 1999, 180(6):1957-1968.

16. Centeno-Lima S, Silveira H, Casimiro C, Aguiar P, do Rosario VE: Kinetics of cytokine expression in mice with invasive aspergillosis: lethal infection and protection. FEMS Immunol Med Microbiol 2002, 32(2):167-173.

17. Ito Jl: T cell immunity and vaccines against invasive fungal diseases. Immunol Invest 2011, 40(7-8):825-838.

18. Stuehler C, Khanna N, Bozza S, Zelante T, Moretti S, Kruhm M, Lurati S, Conrad B, Worschech E, Stevanovic S, Krappmann S, Einsele H, Latgé JP, 
Loeffler J, Romani L, Topp MS: Cross-protective TH1 immunity against Aspergillus fumigatus and Candida albicans. Blood 2011, 117(22):5881-5891.

19. Schroder K, Hertzog PJ, Ravasi T, Hume DA: Interferon-gamma: an overview of signals, mechanisms and functions. J Leukoc Biol 2004, 75(2):163-189.

20. Lehrnbecher T, Tramsen L, Koehl U, Schmidt S, Bochennek K, Klingebiel T: Immunotherapy against invasive fungal diseases in stem cell transplant recipients. Immunol Invest 2011, 40(7-8):839-852.

21. Stevens DA, Brummer E, Clemons KV: Interferon- gamma as an antifungal. $J$ Infect Dis 2006, 194(Suppl 1):S33-S37.

22. Group TICGDCS: A controlled trial of interferon gamma to prevent infection in chronic granulomatous disease. N Engl J Med 1991, 324(8):509-516.

23. Riddell LA, Pinching AJ, Hill S, Ng TT, Arbe E, Lapham GP, Ash S, Hillman R, Tchamouroff S, Denning DW, Parkin JM: A phase III study of recombinant human interferon gamma to prevent opportunistic infections in advanced HIV disease. AIDS Res Hum Retroviruses 2001, 17(9):789-797.

24. Bodasing N, Seaton RA, Shankland GS, Pithie A: Gamma-interferon treatment for resistant oropharyngeal candidiasis in an HIV-positive patient. J Antimicrob Chemother 2002, 50(5):765-766.

25. Jarvis JN, Meintjes G, Rebe K, Williams GN, Bicanic T, Williams A, Schutz C, Bekker LG, Wood R, Harrison TS: Adjunctive interferon-gamma immunotherapy for the treatment of HIV-associated cryptococcal meningitis: a randomized controlled trial. Aids 2012, 26(9):1105-1113.

26. Poynton $\mathrm{CH}$, Barnes RA, Rees J: Interferon gamma and granulocytemacrophage colony-stimulating factor for the treatment of hepatosplenic candidosis in patients with acute leukemia. Clin Infect Dis 1998, 26(1):239-240.

27. Dignani MC, Rex JH, Chan KW, Dow G, deMagalhaes-Silverman M, Maddox A, Walsh T, Anaissie E: Immunomodulation with interferon-gamma and colony-stimulating factors for refractory fungal infections in patients with leukemia. Cancer 2005, 104(1):199-204.

28. Armstrong-James D, Teo IA, Shrivastava S, Petrou MA, Taube D, Dorling A, Shaunak S: Exogenous interferon-gamma immunotherapy for invasive fungal infections in kidney transplant patients. Am J Transplant 2010, 10(8):1796-1803

29. Pappas PG, Kauffman CA, Andes D, Benjamin DK Jr, Calandra TF, Edwards JE Jr, Filler SG, Fisher JF, Kullberg BJ, Ostrosky-Zeichner L, Reboli AC, Rex JH, Walsh TJ, Sobel JD, Infectious Diseases Society of America: Clinical practice guidelines for the management of candidiasis: 2009 update by the Infectious Diseases Society of America. Clin Infect Dis 2009, 48(5):503-535.

30. Oude Lashof AJ, JJWM, Meis JFG, Warris A, van 't Wout JW, Natsch S, Van Zanten A, Verweij PE, Kullberg BJ: Optimalisation of the antibiotic policy in the Netherlands XIII. Dutch Working Party on Antibiotic Policy (SWAB) guideline for the treatment of invasive fungal infections. Nederlands tijdschrift voor geneeskunde 2009, 153:A901.

31. Leentjens J, Kox M, Koch RM, Preijers F, Joosten LA, van der Hoeven JG, Netea MG, Pickkers P: Reversal of Immunoparalysis in Humans In Vivo: A Double-Blind, Placebo-controlled, Randomized Pilot Study. Am J Respir Crit Care Med 2012, 186(9):838-845.

32. Netea MG, Warris A, Van der Meer JW, Fenton MJ, Verver-Janssen TJ, Jacobs $L E$, Andresen T, Verweij PE, Kullberg BJ: Aspergillus fumigatus evades immune recognition during germination through loss of toll-like receptor-4-mediated signal transduction. J Infect Dis 2003, 188(2):320-326.

33. van de Veerdonk FL, Marijnissen RJ, Kullberg BJ, Koenen HJ, Cheng SC, Joosten I, van den Berg WB, Williams DL, van der Meer JW, Joosten LA, Netea MG: The macrophage mannose receptor induces IL-17 in response to Candida albicans. Cell Host Microbe 2009, 5(4):329-340.

34. De Pauw B, Walsh TJ, Donnelly JP, Stevens DA, Edwards JE, Calandra T, Pappas PG, Maertens J, Lortholary O, Kauffman CA, Denning DW, Patterson TF, Maschmeyer G, Bille J, Dismukes WE, Herbrecht R, Hope WW, Kibbler CC, Kullberg BJ, Marr KA, Muñoz P, Odds FC, Perfect JR, Restrepo A, Ruhnke M, Segal BH, Sobel JD, Sorrell TC, Viscoli C, Wingard JR, et al: Revised definitions of invasive fungal disease from the European Organization for Research and Treatment of Cancer/Invasive Fungal Infections Cooperative Group and the National Institute of Allergy and Infectious Diseases Mycoses Study Group (EORTC/MSG) Consensus Group. Clin Infect Dis 2008, 46(12):1813-1821.

35. Eyerich S, Wagener J, Wenzel V, Scarponi C, Pennino D, Albanesi C, Schaller M, Behrendt H, Ring J, Schmidt-Weber CB, Cavani A, Mempel M, Traidl-
Hoffmann C, Eyerich K: IL-22 and TNF-alpha represent a key cytokine combination for epidermal integrity during infection with Candida albicans. Eur J Immuno/ 2011, 41(7):1894-1901.

36. Gresnigt MS, Netea MG, van de Veerdonk FL: Pattern recognition receptors and their role in invasive aspergillosis. Ann N Y Acad Sci 2012, 1273(1):60-67.

37. Sainz J, Salas-Alvarado I, Lopez-Fernandez E, Olmedo C, Comino A, Garcia F, Blanco A, Gomez-Lopera S, Oyonarte S, Bueno P, Jurado M: TNFR1 mRNA expression level and TNFR1 gene polymorphisms are predictive markers for susceptibility to develop invasive pulmonary aspergillosis. Int J Immunopathol Pharmacol 2010, 23(2):423-436.

38. Warris A, Bjorneklett A, Gaustad P: Invasive pulmonary aspergillosis associated with infliximab therapy. N Engl J Med 2001, 344(14):1099-1100.

39. Bellocchio S, Montagnoli C, Bozza S, Gaziano R, Rossi G, Mambula SS, Vecchi A, Mantovani A, Levitz SM, Romani L: The contribution of the Toll-like/IL-1 receptor superfamily to innate and adaptive immunity to fungal pathogens in vivo. J Immunol 2004, 172(5):3059-3069.

40. Sainz J, Perez E, Gomez-Lopera S, Jurado M: IL1 gene cluster polymorphisms and its haplotypes may predict the risk to develop invasive pulmonary aspergillosis and modulate C-reactive protein level. J Clin Immunol 2008, 28(5):473-485.

41. De Luca A, Zelante T, D'Angelo C, Zagarella S, Fallarino F, Spreca A, lannitti RG, Bonifazi P, Renauld JC, Bistoni F, Puccetti P, Romani L: IL-22 defines a novel immune pathway of antifungal resistance. Mucosal Immunol 2010, 3(4):361-373.

42. Gessner MA, Werner JL, Lilly LM, Nelson MP, Metz AE, Dunaway CW, Chan YR, Ouyang W, Brown GD, Weaver CT, Steele C: Dectin-1-dependent interleukin-22 contributes to early innate lung defense against Aspergillus fumigatus. Infect Immun 2012, 80(1):410-417.

43. Lin L, Ibrahim AS, Xu X, Farber JM, Avanesian V, Baquir B, Fu Y, French SW, Edwards JE Jr, Spellberg B: Th1-Th17 cells mediate protective adaptive immunity against Staphylococcus aureus and Candida albicans infection in mice. PLoS Pathog 2009, 5(12):e1000703.

44. Milner JD, Brenchley JM, Laurence A, Freeman AF, Hill BJ, Elias KM, Kanno Y, Spalding C, Elloumi HZ, Paulson ML, Davis J, Hsu A, Asher Al, O'Shea J, Holland SM, Paul WE, Douek DC: Impaired T(H)17 cell differentiation in subjects with autosomal dominant hyper-lgE syndrome. Nature 2008, 452(7188):773-776.

45. Smeekens SP, Henriet SS, Gresnigt MS, Joosten LA, Hermans PW, Netea MG, Warris $A$, van de Veerdonk FL: Low interleukin-17A production in response to fungal pathogens in patients with chronic granulomatous disease. J Interferon Cytokine Res 2012, 32(4):159-168.

46. Saulsbury FT: Successful treatment of aspergillus brain abscess with itraconazole and interferon-gamma in a patient with chronic granulomatous disease. Clin Infect Dis 2001, 32(10):E137-E139.

47. Pasic S, Abinun M, Pistignjat B, Vlajic B, Rakic J, Sarjanovic L, Ostojic N: Aspergillus osteomyelitis in chronic granulomatous disease: treatment with recombinant gamma-interferon and itraconazole. Pediatr Infect Dis J 1996, 15(9):833-834.

48. Malmvall BE, Follin P: Successful interferon-gamma therapy in a chronic granulomatous disease (CGD) patient suffering from Staphylococcus aureus hepatic abscess and invasive Candida albicans infection. Scand J Infect Dis 1993, 25(1):61-66.

49. Ellis M, Watson R, McNabb A, Lukic ML, Nork M: Massive intracerebral aspergillosis responding to combination high dose liposomal amphotericin B and cytokine therapy without surgery. J Med Microbiol 2002, 51(1):70-75.

50. Kelleher $P$, Goodsall A, Mulgirigama A, Kunst $H$, Henderson DC, Wilson $R$, Newman-Taylor A, Levin M: Interferon-gamma therapy in two patients with progressive chronic pulmonary aspergillosis. Eur Respir J 2006, 27(6):1307-1310.

51. Netea MG, Brouwer AE, Hoogendoorn EH, Van der Meer JW, Koolen M, Verweij PE, Kullberg BJ: Two patients with cryptococcal meningitis and idiopathic CD4 lymphopenia: defective cytokine production and reversal by recombinant interferon- gamma therapy. Clin Infect Dis 2004, 39(9):e83-e87.

52. Pappas PG, Rex JH, Lee J, Hamill RJ, Larsen RA, Powderly W, Kauffman CA, Hyslop N, Mangino JE, Chapman S, Horowitz HW, Edwards JE, Dismukes WE, NIAID Mycoses Study Group: A prospective observational study of candidemia: epidemiology, therapy, and influences on mortality in hospitalized adult and pediatric patients. Clin Infect Dis 2003, 37(5):634-643. 
53. Leentjens J, Kox M, van der Hoeven JG, Netea MG, Pickkers P: Immunotherapy for the adjunctive treatment of sepsis: from immunosuppression to immunostimulation. Time for a paradigm change? Am J Respir Crit Care Med 2013, 187(12):1287-1293. doi:10.1164/ rccm.201301-0036CP.

54. Docke WD, Randow F, Syrbe U, Krausch D, Asadullah K, Reinke P, Volk HD, Kox W: Monocyte deactivation in septic patients: restoration by IFN-gamma treatment. Nat Med 1997, 3(6):678-681.

55. Carson WF, Cavassani KA, Dou Y, Kunkel SL: Epigenetic regulation of immune cell functions during post-septic immunosuppression. Epigenetics 2011, 6(3):273-283.

56. Armstrong-James D, Teo I, Herbst S, Petrou M, Shiu KY, McLean A, Taube D, Dorling A, Shaunak S: Renal allograft recipients fail to increase interferongamma during invasive fungal diseases. Am J Transplant 2012, 12(12):3437-3440.

57. Turrel-Davin F, Venet F, Monnin C, Barbalat V, Cerrato E, Pachot A, Lepape A, Alberti-Segui C, Monneret G: mRNA-based approach to monitor recombinant gamma-interferon restoration of LPS-induced endotoxin tolerance. Crit Care 2011, 15(5):R252

58. Chen J, Ivashkiv LB: IFN-gamma abrogates endotoxin tolerance by facilitating Toll-like receptor-induced chromatin remodeling. Proc Natl Acad Sci U S A 2010, 107(45):19438-19443.

59. Xiong $Y$, Medvedev AE: Induction of endotoxin tolerance in vivo inhibits activation of IRAK4 and increases negative regulators IRAK-M, SHIP-1, and A20. J Leukoc Biol 2011, 90(6):1141-1148.

60. Tortorano AM, Kibbler C, Peman J, Bernhardt H, Klingspor L, Grillot R Candidaemia in Europe: epidemiology and resistance. Int J Antimicrob Agents 2006, 27(5):359-366.

doi:10.1186/1471-2334-14-166

Cite this article as: Delsing et al: Interferon-gamma as adjunctive immunotherapy for invasive fungal infections: a case series. $B M C$ Infectious Diseases 2014 14:166.

\section{Submit your next manuscript to BioMed Central and take full advantage of:}

- Convenient online submission

- Thorough peer review

- No space constraints or color figure charges

- Immediate publication on acceptance

- Inclusion in PubMed, CAS, Scopus and Google Scholar

- Research which is freely available for redistribution 